\title{
Lipoblastoma Mimicking Myxoid Liposarcoma: A Clinical Report and Literature Review
}

\author{
Akihito Nagano, ${ }^{1}$ Takatoshi Ohno, ${ }^{1}$ Yutaka Nishimoto, ${ }^{1}$ Yoshinobu Hirose, ${ }^{2}$ \\ Satoru Miyake $^{1}$ and Katsuji Shimizu ${ }^{1}$
}

\author{
${ }^{1}$ Department of Orthopedic Surgery, Graduate school of Medicine, Gifu University, Gifu, Japan \\ ${ }^{2}$ Department of Tumor Pathology, Graduate School of Medicine, Gifu University, Gifu, Japan
}

Lipoblastoma is an uncommon benign lipomatous tumor, occurring typically in children less than 3 years of age. The magnetic resonance image (MRI) is a useful tool for diagnosis of lipoblastoma; its imaging typically shows high-intensity signals on both T1-weighted (T1-W) and T2 weighted (T2-W) images. Here, we present a 12-year-old female patient with a painless mass on the anterior right shoulder. MRI showed the mass with low-intensity signals on T1-W and high-intensity signals on T2-W images. Because of the atypical age and MRI findings, it was difficult to make a conclusive diagnosis of the tumor as lipoblastoma preoperatively. Histopathological examination of the excised tumor showed spindle-shaped or stellate cells embedded in the myxoid matrix, and a few small irregular clusters of mature fat cells that are separated by connective tissue septa. There were some immature, lipoblast-like cells dispersed. These findings are consistent with lipoblastoma, and myxoid liposarcoma was considered as one of the differential diagnosis. We finally diagnosed the tumor as a lipoblastoma for the reasons that there were many mature fat cells and no atypical cells for a myxoid liposarcoma. The postoperative course was uneventful and no recurrence was observed 5 years after the operation. The patient presented is worthy of note due to the unusual characteristics of the tumor. Even in the case of adolescent or older patients with atypical imaging, lipoblastoma should be considered as one of differential diagnosis.

Keywords: lipoblastoma; neoplasm; magnetic resonance imaging; adipose tumor; soft tissue tumor Tohoku J. Exp. Med., 2011, 223 (1), 75-78. C 2011 Tohoku University Medical Press

Lipoblastoma is a rare benign soft-tissue childhood tumor occurring most commonly in children less than 3 years of age (Chung and Enzinger 1973; Mentzel et al. 1993), and magnetic resonance image (MRI) is a useful tool for its diagnosis and preoperative evaluation (Reiseter et al. 1999). MRIs of this type of tumor are typically characterized by high-intensity signals on both $\mathrm{T} 1$ weighted (T1-W) and T2 weighted (T2-W) images (Letourneau et al. 1993). We report a 12-year-old female patient with lipoblastoma in the shoulder, which was difficult to diagnose preoperatively because of the age and its atypical MRI findings. Meanwhile, the presented tumor showed generally low-intensity signals on $\mathrm{T} 1-\mathrm{W}$, and high-intensity signals on $\mathrm{T} 2-\mathrm{W}$ images. These findings were not consistent with lipomatous tumor, and finally we made a diagnosis considering all findings obtained.

\section{Clinical Findings}

A 12-year-old female was referred to our clinic with a 3-month history of a painless mass on the anterior right shoulder. The patient had no history of trauma or constitu- tional symptoms. Physical examination showed a $6 \times 3 \mathrm{~cm}$ elastic soft mass on the anteromedial aspect of the right humerus. The mass was non-tender, well circumscribed, and distinct from surrounding tissue, and did not pulsate. The overlying skin showed none of reddening, increased warmth and vein distention. Neurological examination and pulses of the upper limbs were normal. Results of laboratory tests were within normal limits.

Plain radiography of the right shoulder showed no abnormal calcifications and no abnormalities of the humerus. MRI showed that the mass was well defined and located between the deltoid and greater pectoral muscles (Fig. 1). The mass generally showed low-intensity signals on T1-W and high-intensity signals on T2-W images. There were areas that showed high-intensity signals on T1-W, and low-intensity signals on $\mathrm{T} 2-\mathrm{W}$ images. With fat-suppression sequences using short T1 inversion recovery (STIR), the mass showed generally high-intensity signals. After intravenous administration of a gadolinium-based contrast material, the tumor image was weakly enhanced. Thallium scintigraphy showed no intense uptake in the tumor.

Received September 10, 2010; revision accepted for publication December 16, 2010. doi: 10.1620/tjem.223.75

Correspondence: Takatoshi Ohno, M.D., Ph.D., Department of Orthopaedic Surgery, Gifu University School of Medicine, 1-1 Yanagido, Gifu 501-1194, Japan.

e-mail: takaohno@gifu-u.ac.jp 


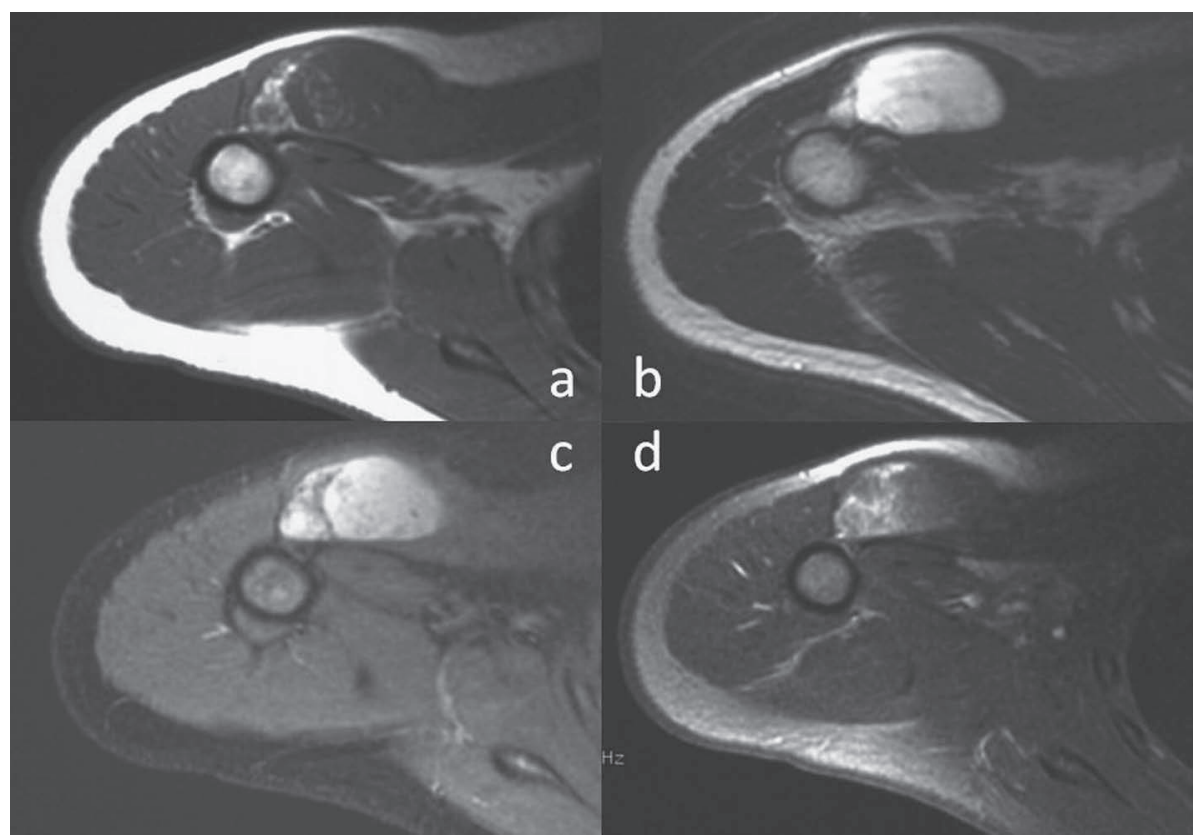

Fig. 1. MRI of the right shoulder of a 12 year-old female.

a. T1-weighted axial image showing an intra-muscular encapsulated mass. The mass is almost homogenous with lowintensity signals and contains a few high-intensity regions.

b. T2-weighted axial section image. The mass shows mostly high-intensity signals.

c. STIR axial section showing no suppression of the signal intensity of the mass, while subcutaneous fat areas are well suppressed.

d. T1-weighted Gd-DPTA-enhanced axial section showing heterogeneous enhancement of the mass.

Therefore, it was considered that alternative diagnoses include hemorrhage after rupture of muscles, myxoma, neurinoma as a benign tumor, and myxoid liposarcoma as a malignant tumor.

Due to the findings above, our clinical impression was that it was not a lipomatous tumor.

A biopsy was considered essential to make a definite diagnosis. An excisional biopsy was chosen because it could preserve the neurovascular band in case of additional resection if the tumor turned out to be malignant.

During the operation, the deltoid muscle was split and an incision was made directly into the tumor. There was no invasion found into any of the surrounding tissue, and the mass was completely excised. Macroscopically, the mass was well-circumscribed, elastic soft, yellowish and homogenous.

Histopathological examination showed the tumor to be well encapsulated. Spindle-shaped or stellate cells were embedded in the myxoid matrix, and a few small irregular clusters of mature fat cells were separated by connective tissue septa of varying thickness. There was a variable degree of cellular differentiation, and vascularization was clearly evident. There were some immature, lipoblast-like cells and mesenchmal cells dispersed throughout (Fig. 2). Ultrastructurally, the tumor cells were irregularly stellate, and cytoplasmic projections were observed engulfing the surrounding myxoid stroma, resulting in a vacuolated appearance. The cytoplasm was rich in filaments, contained

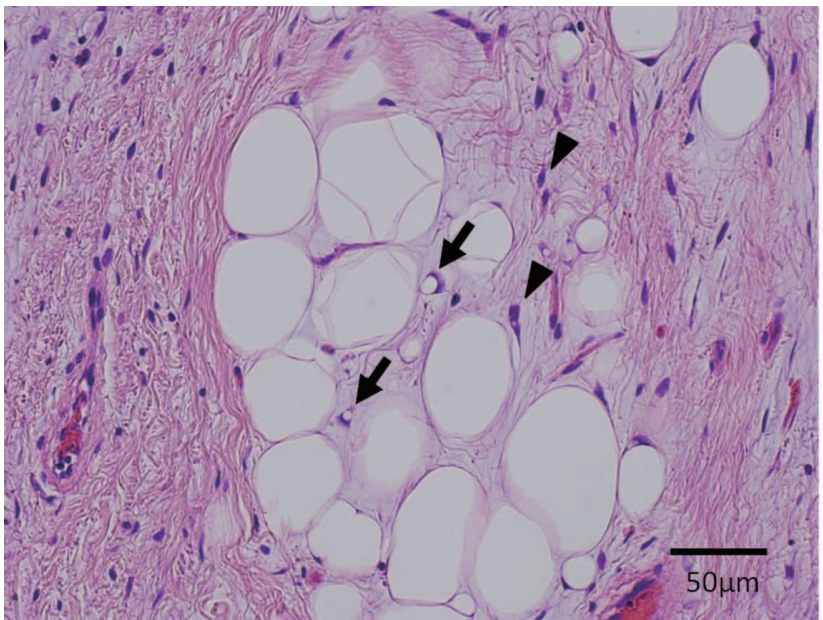

Fig. 2. The tumor section stained with Hematoxylin and eosin.

Shown are a few small irregular clusters of mature fat cells that are separated by connective tissue septa. Some lipoblast-like cells and mesenchymal cells are indicated with arrows and arrowheads, respectively. Atypical cells are not present. $(\times 40)$

small vesicles, which were focally clustered, and also contained vacuoles. A few cells possessed fat droplets (Fig. 3).

These features were consistent with lipoblastoma, and myxoid liposarcoma is considered as one of the differential diagnosis. Finally, we diagnosed the tumor as a lipoblas- 


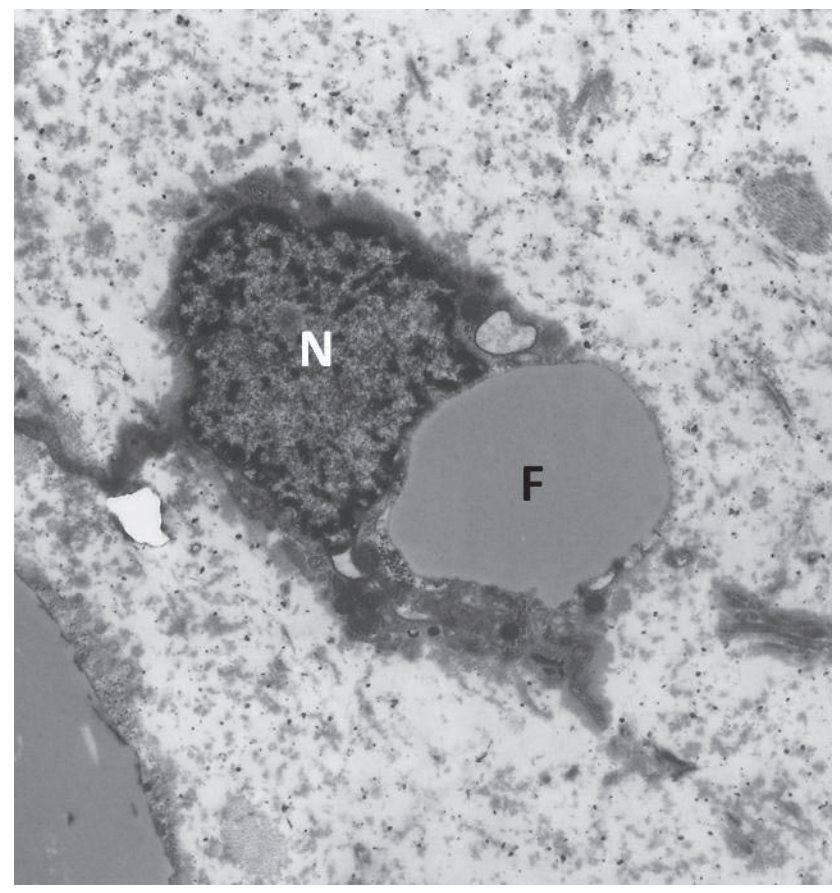

Fig. 3. Electron micrograph of the tumor.

A tumor cell presented in this picture contains fat droplet (N: nucleus, F: fat droplet).

toma for the reason that there were many mature fat cells, no atypical cells and small amount of vascularization for a myxoid liposarcoma. Furthermore, the lobulated appearance of the tumor, which is characteristic of lipoblastomas, supported this diagnosis. The postoperative course was uneventful and no recurrence was observed 5 years after the operation. The patient and her family have provided permission to publish these features of her case, and the identity of the patient has been protected.

\section{Discussion}

Lipoblastoma is a rare, benign neoplasm that consists of immature fat cells at various degrees of maturity and occurs exclusively in childhood, with a male predominance between 1.5 and 3:1 (Chung and Enzinger 1973; Stringel et al. 1982). It has a good prognosis, and does not behave aggressively or metastasize, and therefore the treatment of choice is complete but conservative excision (Chung and Enzinger 1973; Mentzel et al. 1993; Coffin 1994; Gilbert et al. 1996). This type of tumor is rare in patients older than 8 years, however, it has been reported in a number of patients over 10 years of age (Reinders et al. 1983; Jimenez 1986; Gisselsson et al. 2001). In the present report, the patient was 12 years old and was considered relatively old for the condition, and hence it was considered to be rare. (Table 1)

Pathological features of lipoblastoma include a wide spectrum of adipocytic differentiation, in which mono- and multivacuolated lipoblasts are admixed with mature adipocytes. The adipocytic cells are often set in a variably myxoid matrix containing spindle or stellate mesenchymal cells,
Table 1. A review of the age of lipoblastoma cases.

\begin{tabular}{lcccc}
\hline & year & cases & $\begin{array}{c}\text { age } \\
\text { (mo.) }\end{array}$ & $\begin{array}{c}\text { mean } \\
\text { (mo.) }\end{array}$ \\
\hline Chung \& Enzinger & 1973 & 35 & $0-84$ & 12 \\
Mentzel et al. & 1993 & 14 & $0-72$ & 32.4 \\
Hicks et al. & 2001 & 25 & $2-120$ & 20 \\
Chun et al. & 2001 & 7 & $3-29$ & 15.6 \\
Gisselsson et al. & 2001 & 16 & $8-156$ & 45.1 \\
Jung SM et al. & 2005 & 16 & $5-49$ & 11.5 \\
Moholkar S et al. & 2006 & 12 & $6-72$ & 18 \\
Present patient & 2010 & 1 & 144 & - \\
\hline
\end{tabular}

mo., months.

and the tumor is usually organized into lobules, separated by fibrous septae (Weiss and Goldblum 2008).

The ultrastructural features of lipoblastoma are variable. As in normal developing fat, the cells show a wide morphologic spectrum ranging from immature mesenchymal cells and preadipocytes to maltivacuolar lipocytes. The lipoblasts contain numerous vesicles, round to oval mitochondria, and well-developed Golgi membranes. The present patient showed that the tumor cells contained small vesicles and vacuoles. A few cells possessed fat droplets as a mature adipocyte shows. And these cells were observed engulfing the surrounding myxoid stroma, which is compatible with the features of both lipoblastoma and mixoid liposarcoma. In fact, it is reported the principal differential diagnostic consideration of lipoblastoma is mixoid liposarcoma (Weiss and Goldblum 2008).

The origin of this disease is still unknown; however, adipocytic progenitor cells are considered to be one of the candidates of lipoblastoma. Gisselsson et al. (2001) reported chromosomal rearrangements involving band $8 \mathrm{q} 12$ in four lipoblastomas. Each of these alterations targeted the PLAG1 oncogene, which encodes a zinc-finger transcription factor, expressed primarily in fetal tissues and only at very low levels postnatally. They propounded a possible mechanism of tumorigenesis of the disease is that the presence of PLAG1 overexpression transforms mesenchymal progenitor cells to lipoblastoma cells, with various degrees of proliferation and differentiation (Gisselsson et al. 2001). It might be an explanation why this disease occurs in younger age, because such progenitor cells are only present in the first few years of life (Gisselsson et al. 2001).

To achieve a diagnosis, the patient's age, gender, the location of the tumor, radiological features, and histological findings are of some help. However, diagnosis in somewhat older patients can be problematic and, to a degree, arbitrary (Mentzel et al. 1993). Mentzel et al. (1993) presented a patient diagnosed with myxoid liposarcoma that could be called a lipoblastoma if the patient had been 10 years younger. In the present report, the patient age was relatively old and it was difficult to make a differential diagnosis between myxoid liposarcoma and lipoblastoma. 
Reiseter et al. (1999) reported that MRI is an essential tool for diagnosis and preoperative evaluation of lipoblastoma. MRI can show anatomical detail, which is essential for successful radical tumor excision. Also, in general, although lipoblastoma mostly gives a high-intensity signal on both T1-W and T2-W images, it may be hypointense and more heterogeneous with subcutaneous fat on the T1-W image (Letourneau et al. 1993; Gilbert et al. 1996). However, in the present patient, the mass was found to be generally homogenous with low-intensity signals on T1-W, and with high-intensity signals on $\mathrm{T} 2-\mathrm{W}$ images. Furthermore, with fat-suppression sequences, the mass showed generally high-intensity signals. These findings are non-specific, but they are not consistent with lipomatous tumors. In this patient, it is possible that the tumor contained a mucinous matrix, resulting in generally low-intensity signals on the T1-W image. Thus, it should be noted that lipoblastoma could show atypical MRI findings.

\section{Conclusion}

We present a patient with lipoblastoma that shows unusual MRI findings.

\section{References}

Chung, E.B. \& Enzinger, F.M. (1973) Benign lipoblastomatosis. An analysis of 35 cases. Cancer, 32, 482-492.

Chun, Y.S., Kim, W.K., Park, K.W., Lee, S.C. \& Jung S.E. (2001) Lipoblastoma. J. Pediatr. Surg., 36, 905-907.

Coffin, C.M. (1994) Lipoblastoma: an embryonal tumor of soft tissue related to organogenesis. Semin. Diagn. Pathol., 11, 98-103.

Hicks, J., Dilley, A., Patel, D., Barrish, J., Zhu, S.H. \& Brandt, M.
(2001) Lipoblastoma and lipoblastomatosis in infancy and childhood: histopathologic, ultrastructural, and cytogenetic features. Ultrastruct. Pathol., 25, 321-333.

Gilbert, T.J., Goswitz, J.J., Teynor, J.T. \& Griffiths, H.J. (1996) Lipoblastoma of the foot. Skeletal Radiol., 25, 283-286.

Gisselsson, D., Hibbard, M.K., Dal Cin, P., Sciot, R., His, B.L., Kozakewich, H.P. \& Fletcher, J.A. (2001) PLAG1 alterations in lipoblastoma: involvement in varied mesenchymal cell types and evidence for alternative oncogenic mechanisms. Am. J. Pathol., 159, 955-962.

Jimenez, J.F. (1986) Lipoblastoma in infancy and childhood. J. Surg. Oncol., 32, 238-244.

Jung, S.M., Chang, P.Y., Luo, C.C., Huang, C.S., Lai, J.Y. \& Hsueh, C. (2005) Lipoblastoma/ lipoblastomatosis: a clinicopathologic study of 16 cases in Taiwan. Pediatr. Surg. Int., 21, 809-812.

Letourneau, L., Dufour, M. \& Deschenes, J. (1993) Shoulder lipoblastoma: magnetic resonance imaging characteristics. Can. Assoc. Radiol. J., 44, 211-214.

Mentzel, T., Calonje, E. \& Fletcher, C.D. (1993) Lipoblastoma and lipoblastomatosis: a clinicopathological study of 14 cases. Histopathology, 23, 527-533.

Moholkar, S., Sebire, N.J. \& Roebuck, D.J. (2006) Radiologicalpathological correlation in lipoblastoma and lipoblastomatosis. Pediatr. Radiol., 36, 851-856.

Reinders, J., Noyez, L., Munting, J. \& van den Tweel, J. (1983) A benign inguinal lipoblastoma in a 14 -year-old girl. A case report. Acta Chir. Belg., 83, 427-429.

Reiseter, T., Nordshus, T., Borthne, A., Roald, B., Naess, P. \& Schistad, O. (1999) Lipoblastoma: MRI appearances of a rare paediatric soft tissue tumour. Pediatr. Radiol., 29, 542-545.

Stringel, G., Shandling, B., Mancer, K. \& Ein, S.H. (1982) Lipoblastoma in infants and children. J. Pediatr. Surg., 17, 277-280.

Weiss, S.W. \& Goldblum, J.R. (2008) Enzinger and Weiss's SOFT TISSUE TUMORS, $5^{\text {th }}$ ed., Mosby, Philadelphia, PA, pp. 452-456. 